\title{
Repurposing Dupilumab May Treat Advanced COVID-19 Pa- tients With Severe Acute Respi- ratory Syndrome By Mitigating Cytokine Storm
}

\author{
Bronnil Hawill ${ }^{1}$ and Joseph Geraci ${ }^{2}$ \\ ${ }^{1}$ Charles University, Second Faculty of Medicine, Prague, Czechia \\ ${ }^{2}$ NetraMark Corp, Toronto, Canada \\ ${ }^{2}$ Queen's University, Molecular Med, Kingston, Ontario, Canada
}

March 18, 2020

\section{Abstract}

ARS-CoV-02, or more popularly known as COVID-19, is a positive-sense RNA virus that is currently the cause of a pandemic. Due to its highly contagious nature, many countries are closing their borders and placing populations in quarantine in order to slow down transmission. The sharp surge in demand for immediate care units are leaving hospitals depleted of resources. Drug repositioning is the attempt at using an already approved drug to create a favorable phenotype to a disease it was not originally intended for. Drug repositioning is the quickest and safest way to treat patients with such limited time.

\section{Introduction}

Children and adults interact with viruses differently, which might be due to a differentiation in immune response to a novel antigen. The age group that handles the virus most effectively are ages 10 and under, which is approximately the age the thymus stops growing and begins to regress ${ }^{1}$. The thymus is an organ that allows t- lymphocytes to gain immunocompetence. A subgroup of these same t-lymphocytes are shown to cause an immune response, known as a cytokine storm. This cytokine storm is heavily linked with a poor prognosis in SARS patients ${ }^{2}$, especially in elderly patients. By targeting this mechanism we propose a repositioned drug that targets the IL-4 and IL-13 complex, which is shown to treat type 2 inflammatory diseases ${ }^{3}$.

\subsection{T-Lymphocyte function}

T-lymphocytes are a subgroup of immune cells that are involved in cell mediated immunity. They travel from bone marrow, as progenitor T-lymphocytes, to the thymus where they achieve immunocompetence and undergo a Positive and Negative selection. In the thymus they further differentiate to become cytotoxic T-cells, regulatory T-cells, memory cells, or helper Tcells.

Cytotoxic T-lymphocytes circulate in the blood stream looking for distressed cells presenting a MHC I epitope. Once bound, they trigger apoptosis by injecting granzymes into the cytoplasm of the distressed cell. A part of the cytotoxic T-lymphocyte also functions as a 
memory cell.

Helper T-lymphocytes also circulate the blood looking for MHC II antigen presenting cells. Once they bound a MHC II epitope they proliferate and differentiate into either memory or effector cells. These effector cells play a crucial role in initiating a cytokine storm to prepare other immune cells.

\subsection{Subtypes of helper T-lymphocytes}

Helper T-lymphocytes can be further divided into Th1lymphocytes, Th2-lymphocytes, Th17-lymphocytes, and ThF lymphocytes.

Although each has a unique function, we are more interested in the Th2-lymphocytes which are activated with IL-4. IL-4 induces the differentiation of a naive helper $\mathrm{T}$ cell to the Th2 lymphocyte, and through a positive feedback loop, which in return produces IL-4 (see figure 1). This IL-4 loop is the circuitry to warn multiple cells downstream of a potential threat - this is how a cytokine storm can be initiated.

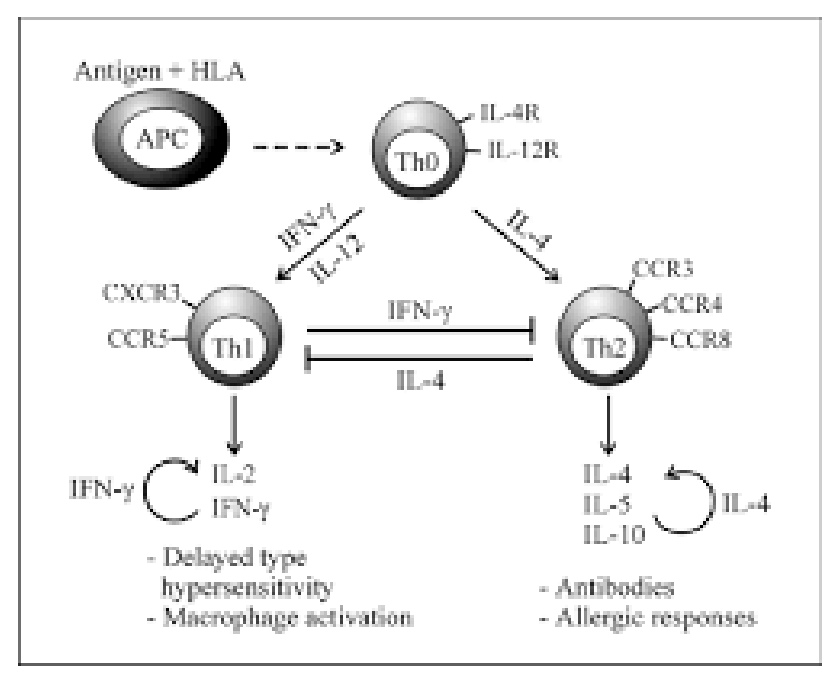

Figure 1: Th2 cell differentiation.

Taken from Westerholm-Ormio, Mia. (2020) ${ }^{4}$

\subsection{NKT lymphocytes}

NKT lymphocytes are an understudied type of lymphocyte which share many characteristics in common with T-lymphocytes and NK cells. We can bring our attention to the fact that the NKT cells in seniors show different downstream reactions when binding to a pathogen ${ }^{5}$. These changes from interferon gamma (IFN-gamma) to gamma delta $\mathrm{T}$ cells induce an extra production in IL-4 in the elderly, where IL-4 is shown to create a cytokine storm.

\subsection{Cytokine storm and IL-4}

IL-4 is an overlooked target, but once we elucidate the understudied functions of IL-4 we begin to justify a strong trend in IL-4 expression and severity in the cytokine storm. Studies have shown IL-4 to induce a secondary hemophagocytic lymphohistiocytosis (HLH), which is a cytokine storm syndrome that is not dependant on $\mathrm{T}$ cells or interferon gamma ${ }^{6}$, suggesting that IL-4 alone is capable of creating secondary HLH.

\subsection{Th2-lymphocyte inflammation path- way}

Th2-lymphocytes and B-lymphocytes work synergistically, causing the B-lymphocytes to differentiate into either memory or anti-body producing effector cells. This reaction causes a downstream effect of elevated eosinophilic and basophilic granulocytes. This reaction may further spiral out of control to lead to a pathogenic allergic type I hypersensitivity. The basophilic granulocytes during allergic responses produce IgE, which in turn increases the inflammatory response. The serum IgE activates basophils through a ligand receptor mechanism and results in massive vasodilation of the surrounding blood vessels.

\subsection{COVID-19 HRCT scan comparison}

Note of interest: HRCT scans of both Hypersensitivity Pneumonitis (HP) and COVID-19 patients with respiratory distress show ground-glass opacity due to alveolitis.

\subsection{NKT cells differentiation with age}

Studies show NK and NKT cell functions in immunosenescence differentiate with ageing. NK and NKT cell cytotoxicity decreases with age as well as interferon gamma production by both cells. However, NKT cells with T-cell receptors show gain in cytotoxicity and TCR gamma delta and interferon gamma production.

\subsection{SARS prognosis and IL-4 differenti- ation}

SARS patients with good prognosis had sera levels that conformed to the following pattern: IFNgamma + MCP1 levels were inversely proportional to circulating lymphocyte and monocyte count while neutrophil count was directly proportional to IFNgamma + MCP1 levels. This suggests that high IFNgamma related cytokine storms were related to bad prognosis ${ }^{2}$. Considering the aforementioned, we can see that neutrophils and monocytes come from the same progenitor cell, the CFU-GM which either differentiates to CFU-M (monocyte) or CFU-G (granulocyte). Note that a neutrophil is a granulocyte. The pathway is described in figure 2 
and it suggests that IL-6 enhances the production of CFU-M which would make it an inappropriate target for COVID-19 patients in the advanced stage. However, if it were possible to inhibit G-SCF it would ultimately lower the neutrophil count and increase monocytes relative to each other.

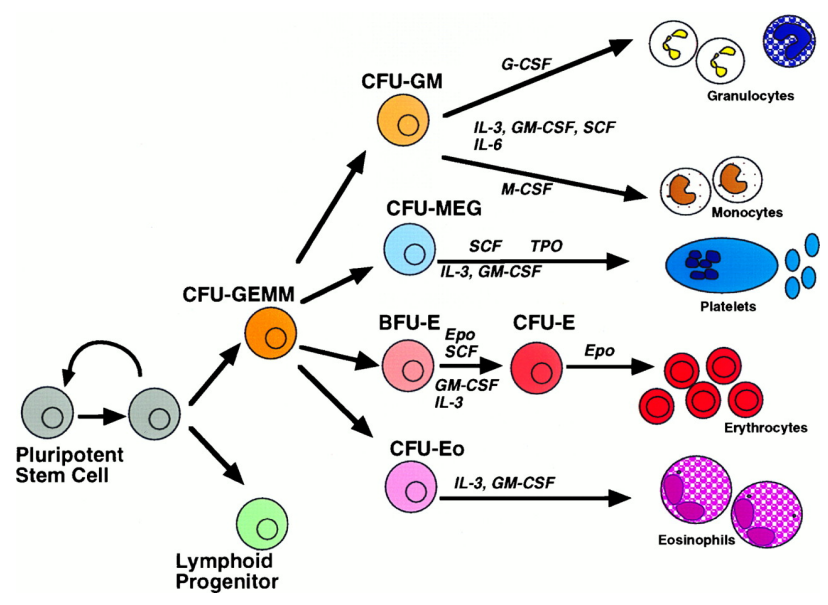

Figure 2: Structure of hematopoietic compartment ${ }^{8}$

Luckily, IL-4 and G-SCF share a common domain and it seems that the receptors differ by only 2 edges see (figure 3). This increases the likelihood that Dupilumab may target the G-SCF receptor and reduce neutrophil production.

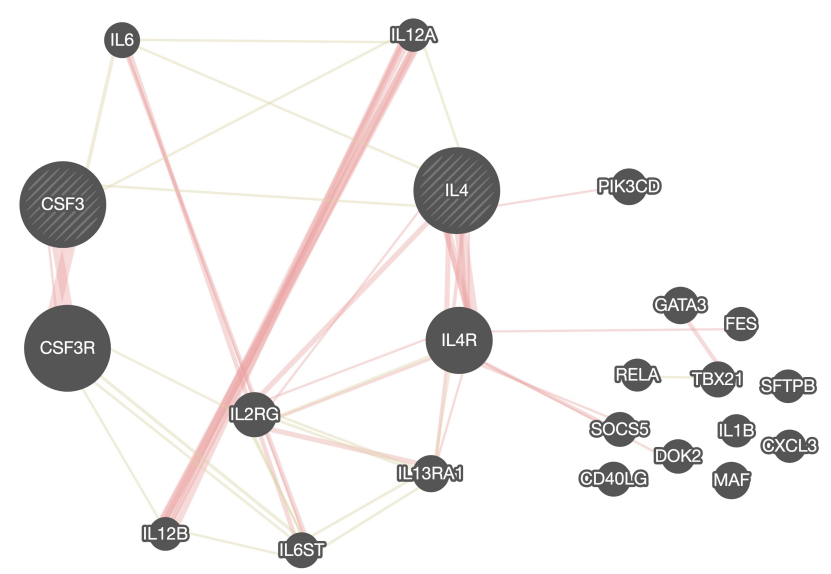

Figure 3: PPI. Red - physical interactions, yellow - shared domain. Created using genemania ${ }^{9}$

\subsection{Targeting NK cells with IL-4}

A new study in 2017 by Ohayon et al., contradicts the popular belief that IL-4 would counter IL-12 production and furthermore inhibit NK cells effector function $^{10}$. They in fact synergize with IL-12 to enhance IFNgamma production. In humans, stimulation of IL-4 or IL-13 produced a dose dependant production of IFNgamma, a major prognostic marker in SARS. It is important to note, that neither IL-4 or IL-13 stimulated IFNgamma production in the absence of IL-12. This suggests a novel drug target, specifically an IL-4 IL-12 antagonist to disturb the synergy.

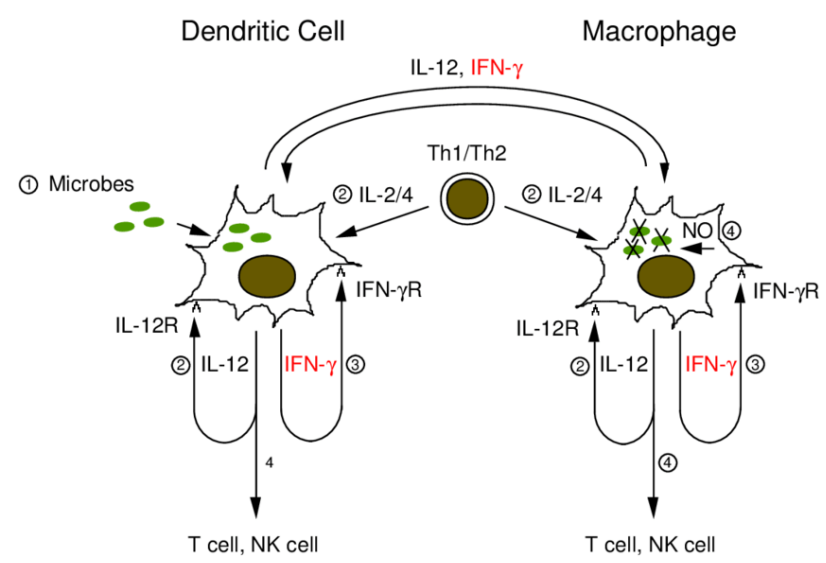

Figure 4: Autocrine APC activation pathway. DC produce IL12 and IFN-gamma ${ }^{11}$

\subsection{NetraAl and how the IL-4 and IL-13 inhibitor for asthma patients was found}

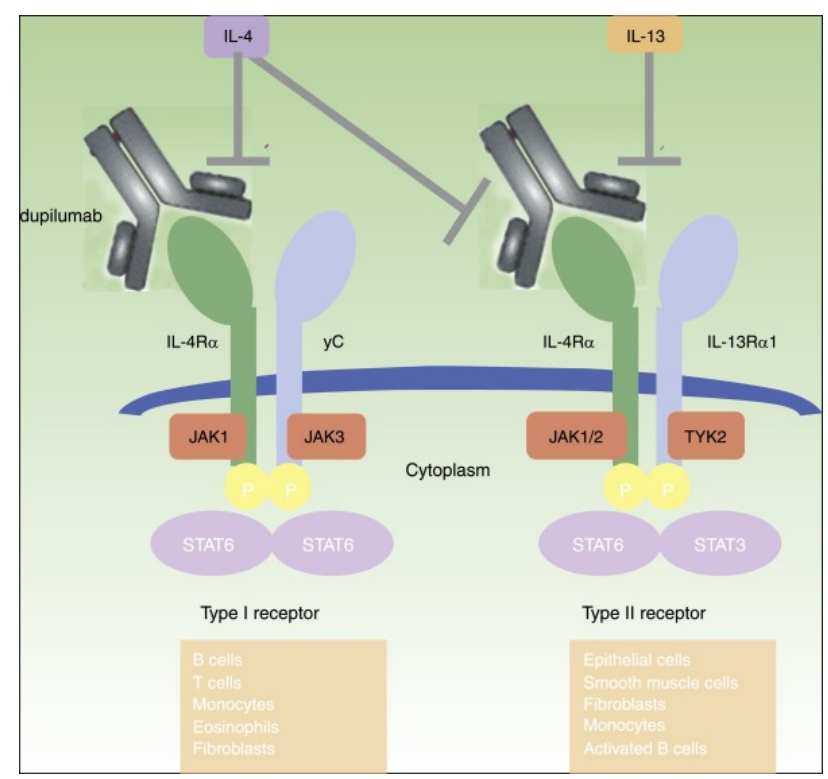

Figure 5: Dupilumab pathway ${ }^{12}$

Dupilumab, a drug tested for patients with uncontrolled, moderate to severe asthma, is an IL-4 and IL-13 antagonist which has proven to reduce severe exacerbation rates and improved forced expiratory volume (FEV1), while reducing type 2 inflammatory biomarkers. The NetraMark Corp's technology called the NetraHealthAtlas, which is part of the NetraAI technology, is a unique machine learning technology that derives its insights by utilizing transcriptomic data from patient populations. As there are no COVID-19 data of this sort available, we used transfer learning by utilizing what the machine learned 
from immune differentiated elderly patients. The NetraHealthAtlas proceeds as follows: it learns from the patient population, finds subpopulations that are differentiated beyond what is understood at the clinically defined level, extracts the genetics involved, builds protein interaction/co-expression networks, utilizes network mathematics to triangulate and find the Achilles heel of the subtype, and then assembles a list of potential drugs that could be utilized. The human intervention here is that we chose to focus on intervening with the cytokine storm and thus led the horse to water, so to speak.

\section{Conclusion}

Here we utilized a unique kind of machine learning technology that has learned from a cohort of elderly gene expression samples, who did not have the COVID19 infection. This reservoir of learning allowed us to transfer what it learned to help us pinpoint a potential mechanism of cytokine storms that could be mitigated by Dupilumab. We suspect that the timing of administration of Dupilumab is critical and could be efficacious during the later stages of the infection so that the cytokine storm could be mediated, especially in older patients. 


\section{References}

1. Minato N. T Cell Senescence and Autoimmunity. In: Nakao K, Minato N, Uemoto S, editors. Innovative Medicine: Basic Research and Development [Internet]. Tokyo: Springer; 2015. Available from: https://www.ncbi.nlm.nih.gov/books/NBK500331/

2. Huang KJ, et al. 2005. An interferon-gammarelated cytokine storm in SARS patients. J. Med. Virol. 75:185-194

3. Licari A, et al. 2020. Dupilumab to Treat Type 2 Inflammatory Diseases in Children and Adolescents. Paediatr Drugs. 2020 Mar 11. doi: 10.1007/s40272-020-00387-2.

4. Westerholm-Ormio, Mia. (2020). Immunologic Inflammation in the Small Intestine of Children : Cytokine Profiles and Immunologic Markers in Potential Coeliac Disease, Type 1 Diabetes, Graftversus-Host Disease, and Food Allergy.

5. Milner JD, Orekov T, Ward JM, et al. Sustained IL-4 exposure leads to a novel pathway for hemophagocytosis, inflammation, and tissue macrophage accumulation. Blood. 2010;116(14):2476-2483. doi:10.1182/blood2009-11-255174

6. Randy Q. Cron and Edward M. Behrens. Cytokine Storm Syndrome. Page 505

7. Mocchegiani E. and Malavolta M. NK and NKT cell functions in immunosenescence. Aging Cell 3(4):177-84

8. Socolovsky M. et al., Control of hematopoietic differentiation: Lack of specificity in signaling by cytokine receptors. PNAS June 9, $1998 \quad 95$ (12) 6573-6575; https://doi.org/10.1073/pnas.95.12.6573

9. Warde-Farley D. et al. The GeneMANIA prediction server: biological network integration for gene prioritization and predicting gene function.

10. Ohayon et al. IL-4 and IL-13 modulate natural killer cell responses under inflammatory conditions. J Immunol May 1, 2017, 198 (1 Supplement) 194.11.

11. Ohteki, T. \& Koyasu, Shigeo. (2001). Role of antigen-presenting cells in innate immune system. Archivum immunologiae et therapiae experimentalis. 49 Suppl 1. S47-52.

12. S. Ferreira and T. Torres. Dupilumab for the Treatment of Atopic Dermatitis. https://doi.org/10.1016/j.adengl.2018.02.019 